\title{
LAGRANGE GEOMETRY ON TANGENT MANIFOLDS
}

\author{
IZU VAISMAN
}

Received 13 March 2003

\begin{abstract}
Lagrange geometry is the geometry of the tensor field defined by the fiberwise Hessian of a nondegenerate Lagrangian function on the total space of a tangent bundle. Finsler geometry is the geometrically most interesting case of Lagrange geometry. In this paper, we study a generalization which consists of replacing the tangent bundle by a general tangent manifold, and the Lagrangian by a family of compatible, local, Lagrangian functions. We give several examples and find the cohomological obstructions to globalization. Then, we extend the connections used in Finsler and Lagrange geometry, while giving an index-free presentation of these connections.
\end{abstract}

2000 Mathematics Subject Classification: 53C15, 53C60.

1. Preliminaries. Lagrange geometry $[3,6,7]$ is the extension of Finsler geometry (e.g., [1]) to transversal "metrics" (nondegenerate quadratic forms) of the vertical foliation (the foliation by fibers) of a tangent bundle, which are defined as the Hessian of a nondegenerate Lagrangian function. In the present paper, we study the generalization of Lagrange geometry to arbitrary tangent manifolds [2]. The locally Lagrange-symplectic manifolds [12] are an important particular case. In this section, we recall various facts about the geometric structures that we need for the generalization. Our framework is the $C^{\infty}$ category, and we will use the Einstein summation convention, where convenient.

First, a leafwise locally affine foliation is a foliation such that the leaves have a given locally affine structure that varies smoothly with the leaf. In a different formulation [10], if $M$ is a manifold of dimension $m=p+q$, a $p$-dimensional leafwise locally affine foliation $\mathscr{F}_{F}$ on $M$ is defined by a maximal, differential, affine atlas $\left\{U_{\alpha}\right\}$, with local coordinates $\left(x_{\alpha}^{a}, y_{\alpha}^{u}\right)(a=1, \ldots, q ; u=1, \ldots, p)$, and transition functions of the local form

$$
x_{\beta}^{a}=x_{\beta}^{a}\left(x_{\alpha}^{b}\right), \quad y_{\beta}^{u}=\sum_{v=1}^{p} A_{(\alpha \beta) v}^{u}\left(x_{\alpha}^{b}\right) y_{\alpha}^{v}+B_{(\alpha \beta)}^{u}\left(x_{\alpha}^{b}\right)
$$

on $U_{\alpha} \cap U_{\beta}$. Then, the leaves of $\mathscr{F}$ are locally defined by $x^{a}=$ const, and their local parallelization is defined by the vector fields $\partial / \partial y^{u}$. Furthermore, if the atlas that defines a leafwise locally affine foliation has a subatlas such that $B_{(\alpha \beta)}^{u}=0$ for its transition functions, the foliation, with the structure defined 
by the subatlas, will be called a vector bundle-type foliation. Notice that, if one such subatlas exists, similar ones are obtained by coordinate changes of the local form

$$
\tilde{x}_{\alpha}^{a}=\tilde{x}_{\alpha}^{a}\left(x_{\alpha}^{b}\right), \quad \tilde{y}_{\alpha}=y_{\alpha}^{a}+\xi_{(\alpha \beta)}^{a}\left(x_{\alpha}^{b}\right) .
$$

For any foliation $\mathscr{F}$, geometric objects of $M$ that either project to the space of leaves or, locally, are pullbacks of objects on the latter are said to be projectable or foliated $[8,9]$. In particular, a foliated bundle is a bundle over $M$ with a locally trivializing atlas with foliated transition functions. The transversal bundle $\nu \mathscr{F}=T M / T \mathscr{F}$ is foliated. Formulas (1.1) show that for a leafwise locally affine foliation $\mathscr{F}$ the tangent bundles $T \mathscr{F}$ and $T M$ are foliated bundles as well. For a foliated bundle, we can define foliated cross sections. Notice that, if $\mathscr{F}$ is a leafwise locally affine foliation, a vector field on $M$ which is tangent to $\mathscr{F}_{F}$ is foliated as a vector field, since it projects to 0 , but it may not be a foliated cross section of $T \mathscr{F}$ !

Furthermore, for a leafwise locally affine foliation, one also has leafwise affine objects which have an affine character with respect to the locally affine structure of the leaves. For instance, a leafwise locally affine function is a function $f \in C^{\infty}(M)$ such that $Y f$ is foliated for any local parallel vector field $Y$ along the leaves of $\mathscr{F}$. With respect to the affine atlas, a leafwise locally affine function has the local expression

$$
f=\sum_{u=1}^{p} \alpha_{u}\left(x^{a}\right) y^{u}+\beta\left(x^{a}\right) .
$$

A leafwise locally affine $k$-form is a $k$-form $\lambda$ such that $i(Z) \lambda=0$ for all the tangent vector fields $Z$ of $\mathscr{F}$ and the Lie derivative $L_{Y} \lambda$ is a foliated $k$-form for all the parallel fields $Y$. Then, $\lambda$ has an expression of the form (1.3) where $\alpha_{u}$, $\beta$ are foliated $k$-forms. A leafwise locally affine vector field is an infinitesimal automorphism of the foliation and of the leafwise affine structure, and has the local expression [10]

$$
X=\sum_{a=1}^{q} \xi^{a}\left(x^{b}\right) \frac{\partial}{\partial x^{a}}+\sum_{u=1}^{p}\left[\sum_{v=1}^{p} \lambda_{v}^{u}\left(x^{b}\right) y^{v}+\mu^{u}\left(x^{b}\right)\right] \frac{\partial}{\partial y^{u}} .
$$

Any foliated vector bundle $V \rightarrow M$ produces a sheaf $\underline{V}$ of germs of differentiable cross sections, and a sheaf $\underline{V}_{p r}$ of germs of foliated cross sections. The corresponding cohomology spaces $H^{k}\left(M, \underline{V}_{p r}\right)$ may be computed by a de Rham type theorem [9]. Namely, let $N \mathscr{F}$ be a complementary (normal) distribution of $T \mathscr{F}$ in $T M$. The decomposition $T M=N \mathscr{F} \oplus T \mathscr{F}$ yields a bigrading of differential forms and tensor fields, and a decomposition of the exterior differential as

$$
d=d_{(1,0)}^{\prime}+d_{(0,1)}^{\prime \prime}+\partial_{(2,-1)} .
$$


The operator $d^{\prime \prime}$ is the exterior differential along the leaves of $\mathscr{F}$, it has square zero and satisfies the Poincaré lemma. Accordingly,

$$
0 \longrightarrow \underline{V}_{p r} \stackrel{\subseteq}{\longrightarrow} \underline{V}_{p r} \otimes_{\Phi} \underline{\Omega}^{(0,0)} \stackrel{d^{\prime \prime}}{\longrightarrow} \underline{V}_{p r} \otimes_{\Phi} \underline{\Omega}^{(0,1)} \stackrel{d^{\prime \prime}}{\longrightarrow} \cdots,
$$

where $\Omega$ denotes spaces of differential forms, $\underline{\Omega}$ is the corresponding sheaf of differentiable germs, and $\Phi$ is the sheaf of germs of foliated functions, is a fine resolution of $\underline{V}_{p r}$.

Furthermore, if $\mathscr{F}$ is leafwise locally affine, one also has the spaces $A^{k}(M, \mathscr{F})$ of leafwise locally affine $k$-forms and the corresponding sheaves of germs $\underline{A}^{k}(M, \mathscr{F})$. These sheaves define interesting cohomology spaces, which may be studied by means of the exact sequences [10]

$$
0 \longrightarrow \underline{\Omega}_{p r}^{(k, 0)} \stackrel{\subseteq}{\longrightarrow} \underline{A}^{k}(M, \mathscr{F}) \stackrel{\pi}{\longrightarrow} \underline{\Omega}_{p r}^{(k, 0)} \otimes_{\Phi} \underline{T}^{* \mathscr{F}} p r \rightarrow 0,
$$

where, for $f$ defined by (1.3), $\pi(f)=\alpha_{u} \otimes\left[d y^{u}\right]$, [ $\left.d y^{u}\right]$ being the projections of $d y^{u}$ on $T^{*} \mathscr{F}$.

It is important to recognize the vector bundle-type foliations among the leafwise locally affine foliations. First, notice that a vector bundle-type foliation possesses a global vector field which may be seen as the leafwise infinitesimal homothety, namely,

$$
E=\sum_{u=1}^{p} y^{u} \frac{\partial}{\partial y^{u}},
$$

called the Euler vector field. In the general leafwise locally affine case, (1.8) only defines local vector fields $E_{\alpha}$ on each coordinate neighborhood $U_{\alpha}$, and the differences $E_{\beta}-E_{\alpha}$ yield a cocycle and a cohomology class $[E](\mathscr{F}) \in H^{1}\left(M, \underline{T \mathscr{F}_{p r}}\right)$, called the linearity obstruction [10]. It follows easily that the leafwise locally affine foliation $\mathscr{F}$ has a vector bundle-type structure if and only if $[E](\mathscr{F})=0$ [10]. With a normal distribution $N \mathscr{F}$, we may use the foliated version of de Rham's theorem, and $[E](\mathscr{F})$ will be represented by the global $T \mathscr{F}$-valued 1form obtained by gluing up the local forms $\left\{d^{\prime \prime} E_{\alpha}\right\}$. Accordingly, $[E](\mathscr{F})=0$ if and only if there exists a global vector field $E$ on $M$, which is tangent to the leaves of $\mathscr{F}$ and such that for all $\alpha$,

$$
\left.E\right|_{U_{\alpha}}=E_{\alpha}+Q_{\alpha}
$$

where $Q_{\alpha}$ are projectable. $E$ is defined up to the addition of a global, projectable cross section of $T \mathscr{F}$, and these vector fields $E$ will be called Euler vector fields. The choice of an Euler vector field $E$ is equivalent with the choice of the vector bundle-type structure of the foliation.

We also recall the following result [10]: the vector bundle-type foliation $\mathscr{F}$ on $M$ is a vector bundle fibration $M \rightarrow N$ if and only if the leaves are simply connected and the flat connections defined by the locally affine structure of the leaves are complete. 
EXAMPLE 1.1. On the torus $\mathbb{T}^{p+q}$ with the Euclidean coordinates $\left(x^{a}, y^{u}\right)$ defined up to translations

$$
\tilde{x}^{a}=x^{a}+h^{a}, \quad \tilde{y}^{u}=y^{u}+k^{u}, \quad h^{a}, k^{u} \in \mathbb{Z},
$$

the foliation $x^{a}=$ const is leafwise locally affine and has the normal bundle $d y^{u}=0$. The linearity obstruction $[E]$ is represented by the form $\sum_{u=1}^{q} d y^{u} \otimes$ $\left(\partial / \partial y^{u}\right)$, which is not $d^{\prime \prime}$-exact. Therefore, $[E] \neq 0$ and $\mathscr{F}$ is not a vector bundletype foliation.

EXAMPLE 1.2. Consider the compact nilmanifold $M(1, p)=\Gamma(1, p) \backslash H(1, p)$, where

$$
H(1, p)=\left\{\left(\begin{array}{ccc}
\operatorname{Id}_{p} & X & Z \\
0 & 1 & y \\
0 & 0 & 1
\end{array}\right) \mid X, Z \in \mathbb{R}^{p}, y \in \mathbb{R}\right\}
$$

is the generalized Heisenberg group, and $\Gamma(1, p)$ is the subgroup of matrices with integer entries. The manifold $M(1, p)$ has an affine atlas with the transition functions

$$
\tilde{x}^{i}=x^{i}+a^{i}, \quad \tilde{y}=y+b, \quad \tilde{z}^{i}=z^{i}+a^{i} y+c^{i},
$$

where $x^{i}, z^{i}(i=1, \ldots, p)$ are the entries of $X, Z$, respectively, and $a^{i}, b, c^{i}$ are integers. Accordingly, the local equations $x^{i}=$ const, $y=$ const define a leafwise locally affine foliation $\mathscr{F}$ of $M$ which, in fact, is a fibration by $p$-dimensional tori over a $(p+1)$-dimensional torus. The manifold $M$ is parallelizable by the global vector fields

$$
\frac{\partial}{\partial x^{i}}, \frac{\partial}{\partial y}+\sum_{i=1}^{p} x^{i} \frac{\partial}{\partial z^{i}}, \frac{\partial}{\partial z^{i}}
$$

and the global 1-forms

$$
d x^{i}, d z^{i}-x^{i} d y, d y
$$

and we see that

$$
\operatorname{span}\left\{\frac{\partial}{\partial x^{i}}, \frac{\partial}{\partial y}+\sum_{i=1}^{p} x^{i} \frac{\partial}{\partial z^{i}}\right\}
$$

may serve as a normal bundle of $\mathscr{F}$. It follows that the linearity obstruction is represented by

$$
\sum_{i=1}^{p}\left(d z^{i}-x^{i} d y\right) \otimes \frac{\partial}{\partial z^{i}}
$$

which is not $d^{\prime \prime}$-exact. Therefore, $\mathscr{F}$ is not a vector bundle-type foliation. 
EXAMPLE 1.3. Take the real Hopf manifold $H^{(p+q)}=S^{p+q-1} \times S^{1}$ seen as $\left(\mathbb{R}^{q} \times \mathbb{R}^{p} \backslash\{0\}\right) / G_{\lambda}$, where $\lambda \in(0,1)$ is constant and $G_{\lambda}$ is the group

$$
\tilde{x}^{a}=\lambda^{n} x^{a}, \quad \tilde{y}^{u}=\lambda^{n} y^{u}, \quad n \in \mathbb{Z},
$$

where $x^{a}, y^{u}$ are the natural coordinates of $\mathbb{R}^{q}$ and $\mathbb{R}^{p}$, respectively. Then, the local equations $x^{a}=$ const define a vector bundle-type foliation, which has the global Euler field $E=\sum_{u=1}^{q} y^{u}\left(\partial / \partial y^{u}\right)$. This example shows that compact manifolds may have vector bundle-type foliations.

EXAMPLE 1.4. Consider the manifold

$$
M^{2 n}=\left[\left(\mathbb{R}^{n} \backslash\{0\}\right) \times \mathbb{R}^{n}\right] / K_{\lambda},
$$

where $\lambda \in(0,1)$ and $K_{\lambda}$ is the cyclic group generated by the transformation

$$
\tilde{x}^{i}=\lambda x^{i}, \quad \tilde{y}^{i}=\lambda y^{i}+(1-\lambda) \frac{x^{i}}{\sqrt{\sum_{j=1}^{n}\left(x^{j}\right)^{2}}}
$$

$(i=1, \ldots, n)$. It is easy to check that the equality

$$
E=\sum_{i=1}^{n} y^{i} \frac{\partial}{\partial y^{i}}-\sum_{i=1}^{n} \frac{x^{i}}{\sqrt{\sum_{j=1}^{n}\left(x^{j}\right)^{2}}} \frac{\partial}{\partial y^{i}}
$$

defines a global vector field on $M$, which has the property of the Euler field for the foliation $x^{i}=$ const Therefore, the latter is a vector bundle-type foliation. The change of coordinates

$$
x^{\prime i}=x^{i}, \quad y^{\prime i}=y^{i}-\frac{x^{i}}{\sqrt{\sum_{j=1}^{n}\left(x^{j}\right)^{2}}}
$$

provides a vector bundle-type atlas, and (1.19) becomes

$$
\tilde{x}^{\prime i}=\lambda x^{i}, \quad \tilde{y}^{\prime i}=\lambda y^{i} .
$$

This shows that $M$ is the tangent bundle of the Hopf manifold $H^{n}$ defined in Example 1.3.

Now, we recall the basics of tangent manifolds [2]. An almost tangent structure on a manifold $M$ is a tensor field $S \in \Gamma$ End(TM) such that

$$
S^{2}=0, \quad \operatorname{im} S=\operatorname{ker} S
$$

In particular, the dimension of $M$ must be even, say $2 n$, and $\operatorname{rank} S=n$. Furthermore, $S$ is a tangent structure if it is integrable, that is, locally $S$ looks like the vertical twisting homomorphism of a tangent bundle. This means that 
there exists an atlas with local coordinate $\left(x^{i}, y^{i}\right)(i=1, \ldots, n)$ such that

$$
S\left(\frac{\partial}{\partial x^{i}}\right)=\frac{\partial}{\partial y^{i}}, \quad S\left(\frac{\partial}{\partial y^{i}}\right)=0
$$

The integrability property is equivalent with the annulation of the Nijenhuis tensor

$$
\mathcal{N}_{S}(X, Y)=[S X, S Y]-S[S X, Y]-S[X, S Y]+S^{2}[X, Y]=0 .
$$

A pair $(M, S)$, where $S$ is a tangent structure, is called a tangent manifold.

On a tangent manifold $(M, S)$, the distribution im $S$ is integrable, and defines the vertical foliation $\mathscr{V}$ with $T \mathscr{V}=\operatorname{im} S$. It is easy to see that the transition functions of the local coordinates of (1.24) are of the local form (1.1) with $q=p=n$ and

$$
A_{(\alpha \beta) j}^{i}=\frac{\partial x_{\beta}^{i}}{\partial x_{\alpha}^{j}} .
$$

Therefore, $\mathscr{V}$ is a leafwise locally affine foliation, and the local parallel vector fields along the leaves are the vector fields of the form $S X$, where $X$ is a foliated vector field. In particular, a tangent manifold has local Euler fields $E_{\alpha}$, and a linearity obstruction $[E] \in H^{1}\left(M, \underline{T}_{\mathcal{V}} p r\right)$. If $[E]=0$, the foliation $\mathscr{V}$ will be a vector bundle-type foliation, and $M$ has global Euler vector fields $E$ defined up to the addition of a foliated cross section of $T \mathscr{V}$. Furthermore, if we fix the vector-bundle type structure by fixing an Euler vector field $E$, the triple $(M, S, E)$ will be called a bundle-type tangent manifold.

Using the general result of [10], we see that a tangent manifold is a tangent bundle if and only if it is a bundle-type tangent manifold and the vertical foliation has simply connected, affinely complete leaves.

EXAMPLE 1.5. The Hopf manifold $H^{2 n}$ of Example 1.3 with $q=p=n$ and $S$ defined by (1.24) is a compact, bundle-type, tangent manifold.

EXAMPLE 1.6. The torus of Example 1.1 with $q=p$ and $S$ of (1.24) is a compact, nonbundle-type, tangent manifold.

EXAMPLE 1.7. The manifold $M(1, p) \times(\mathbb{R} / \mathbb{Z})$, with the coordinates of Example 1.2 and a new coordinate $t$ on $\mathbb{R}$, and with $S$ defined by

$$
S\left(\frac{\partial}{\partial x^{i}}\right)=\frac{\partial}{\partial z^{i}}, \quad S\left(\frac{\partial}{\partial y}\right)=\frac{\partial}{\partial t}, \quad S\left(\frac{\partial}{\partial z^{i}}\right)=0, \quad S\left(\frac{\partial}{\partial t}\right)=0
$$


is a compact, non bundle-type, tangent manifold. The linearity obstruction $[E]$ of this manifold is represented by

$$
\sum_{i=1}^{p}\left(d z^{i}-x^{i} d y\right) \otimes \frac{\partial}{\partial z^{i}}+d t \otimes \frac{\partial}{\partial t},
$$

and $[E] \neq 0$.

Tangent bundles posses second order vector fields (semisprays in [6]), so called because they may be locally expressed by a system of second order, ordinary, differential equations. A priori, such vector fields may be defined on any tangent manifold [13], namely, the vector field $X \in \Gamma T M$ ( $\Gamma$ denotes the space of global cross sections) is of the second order if $\left.S X\right|_{U_{\alpha}}-E_{\alpha}$ is foliated for all $\alpha$. But this condition means that $S X$ is a global Euler vector field, hence, only the bundle-type tangent manifolds can have global second order vector fields.

It is important to point out that, just like on tangent bundles (e.g., [3, 6, 11]), if $(M, S, E)$ is a bundle-type tangent manifold and $X$ is a second order vector field on $M$, the Lie derivative $F=L_{X} S$ defines an almost product structure on $M\left(F^{2}=\mathrm{Id}\right)$, with the associated projectors

$$
V=\frac{1}{2}(\mathrm{Id}+F), \quad H=\frac{1}{2}(\mathrm{Id}-F),
$$

such that $\operatorname{im} V=T^{\mathscr{V}}$ and $\operatorname{im} H$ is a normal distribution $N^{\mathscr{V}}$ of the vertical foliation $\mathscr{V}$.

Finally, we give the following definition.

DEFINITION 1.8. A vector field $X$ on a tangent manifold $(M, S)$ is a tangential infinitesimal automorphism if $L_{X} S=0$ ( $L$ denotes the Lie derivative).

Obviously, a tangential infinitesimal automorphism $X$ preserves the foliation $\mathscr{V}$ and its leafwise affine structure. Therefore, $X$ is a leafwise affine vector field with respect to $\mathscr{V}$. Furthermore, in the bundle-type case, if $E$ is an Euler vector field, $[X, E]$ is a foliated cross section of $T^{\mathscr{V}}$.

2. Locally Lagrange spaces. Lagrange geometry is motivated by physics and, essentially, it is the study of geometric objects and constructions that are transversal to the vertical foliation of a tangent bundle and are associated with a Lagrangian (a name taken from Lagrangian mechanics), that is, a function on the total space of the tangent bundle. (See [6] and the $d$-objects defined there.) Here, we use the same approach for a general tangent manifold $(M, S)$, and we refer to functions on $M$ as global Lagrangians and to functions on open subsets as local Lagrangians. 
If $\mathscr{L}$ is a Lagrangian, the derivatives in the vertical directions yield symmetric tensor fields of $M$ defined by

$$
\left(\operatorname{Hess}_{(k)} \mathscr{L}\right)_{x}\left(X_{1}, \ldots, X_{k}\right)=\left.\left(S \tilde{X}_{k}\right) \cdots\left(S \tilde{X}_{1}\right) \mathscr{L}\right|_{x}, \quad x \in M, X_{i} \in T_{x} M,
$$

where $\tilde{X}_{i}(i=1, \ldots, k)$ are extensions of $X_{i}$ to local, $\mathscr{V}$-foliated, vector fields on $M$. (Of course, the result does not depend on the choice of the extensions $\tilde{X}_{i}$.) $\operatorname{Hess}_{(k)} \mathscr{L}$ is called the $k$-Hessian of $\mathscr{L}$. Notice that definition (2.1) may also be replaced by the recurrence formula

$$
\left(\operatorname{Hess}_{(k)} \mathscr{L}\right)_{\chi}\left(\tilde{X}_{1}, \ldots, \tilde{X}_{k}\right)=\left[L_{S \tilde{X}_{k}}\left(\operatorname{Hess}_{(k-1)} \mathscr{L}\right)\right]_{x}\left(\tilde{X}_{1}, \ldots, \tilde{X}_{k-1}\right),
$$

where the arguments are foliated vector fields.

It is worthwhile to notice the following general property.

Proposition 2.1. For any function $\mathscr{L} \in C^{\infty}(M)$, any tangential infinitesimal automorphism $X$ of the tangent manifold $(M, S)$, and any $k=1,2, \ldots$, one has

$$
\operatorname{Hess}_{(k)}(X \mathscr{L})=L_{X}\left(\operatorname{Hess}_{(k)} \mathscr{L}\right) .
$$

Proof. Proceed by induction on $k$, while evaluating the Hessian of $X \mathscr{L}$ on foliated arguments and using the recurrence formula (2.2).

For $k=1$, we get a 1 -form, say $\theta_{\mathscr{L}}$, and for $k=2$, we get the usual Hessian of $\mathscr{L}$ with respect to the affine vertical coordinates $y^{i}$ (see Section 1 ), hereafter to be denoted by either Hess $\mathscr{L}$ or $\mathfrak{g}_{\mathscr{L}}$. Obviously, $g_{\mathscr{L}}$ vanishes whenever one of the arguments is vertical, hence, it yields a well-defined cross section of the symmetric tensor product $\odot^{2} v^{*} \mathscr{V}\left(v^{\mathscr{V}}=T M / T \mathscr{V}\right)$, which we continue to denote by $g_{\mathscr{L}}$. If $\mathcal{g}_{\mathscr{L}}$ is nondegenerate on the transversal bundle $v \mathscr{F}_{\mathscr{F}}$, the Lagrangian $\mathscr{L}$ is said to be regular and $g_{\mathscr{L}}$ is called a (local) Lagrangian metric. We note that if the domain of $\mathscr{L}$ is connected, the regularity of $\mathscr{L}$ also implies that $g_{\mathscr{L}}$ is of a constant signature. With respect to the local coordinates of (1.24), one has

$$
\theta_{\mathscr{L}}=\frac{\partial \mathscr{L}}{\partial y^{i}} d x^{i}, \quad g_{\mathscr{L}}=\frac{1}{2} \frac{\partial^{2} \mathscr{L}}{\partial y^{i} \partial y^{j}} d x^{i} \odot d x^{j} .
$$

In Lagrangian mechanics, one also defines another geometric object related to a Lagrangian, namely, the differential 2-form

$$
\omega_{\mathscr{L}}=d \theta_{\mathscr{L}}=\frac{\partial^{2} \mathscr{L}}{\partial x^{i} \partial y^{j}} d x^{i} \wedge d x^{j}+\frac{\partial^{2} \mathscr{L}}{\partial y^{i} \partial y^{j}} d y^{i} \wedge d x^{j} .
$$

If $\mathscr{L}$ is a regular Lagrangian, $\omega_{\mathscr{L}}$ is a symplectic form, called the Lagrangian symplectic form.

In $[12,13]$, we studied particular symplectic forms $\Omega$ on a tangent manifold $(M, S)$ that are compatible with the tangent structure $S$ in the sense that

$$
\Omega(X, S Y)=\Omega(Y, S X) .
$$


If this happens, $\Omega$ is called a locally Lagrangian-symplectic form since the compatibility property is equivalent with the existence of an open covering $M=$ $\cup U_{\alpha}$, and of local regular Lagrangian functions $\mathscr{L}_{\alpha}$ on $U_{\alpha}$ such that $\left.\Omega\right|_{U_{\alpha}}=\omega_{\mathscr{L}_{\alpha}}$ for all $\alpha$. On the intersections $U_{\alpha} \cap U_{\beta}$, the local Lagrangians satisfy a compatibility relation of the form

$$
\mathscr{L}_{\beta}-\mathscr{L}_{\alpha}=a\left(\varphi_{(\alpha \beta)}\right)+b_{(\alpha \beta)},
$$

where $\varphi_{(\alpha \beta)}$ is a closed, foliated 1-form, $b_{(\alpha \beta)}$ is a foliated function, and $a(\varphi)=$ $\varphi_{i} y^{i}$ where the local coordinates and components are taken either in $U_{\alpha}$ or in $U_{\beta}$. Furthermore, if it is possible to find a compatible (in the sense of (2.7)) global Lagrangian $\mathscr{L}, \Omega$ is a global Lagrangian symplectic form. Conditions for the existence of a global Lagrangian were given in [12, 13]. In particular, a globally Lagrangian-symplectic manifold $M^{2 n}$ cannot be compact since it has the exact volume form $\omega_{\mathscr{L}}^{n}$.

Following the same idea, we give a new definition.

DEFINITION 2.2. Let $\left(M^{2 n}, S\right)$ be a tangent manifold, and $g \in \Gamma \odot^{2} v^{*} \mathcal{V}$ a nondegenerate tensor field. Then $g$ is a locally Lagrangian metric (structure) on $M$ if there exists an open covering $M=\cup U_{\alpha}$ with local regular Lagrangian functions $\mathscr{L}_{\alpha}$ on $U_{\alpha}$ such that $\left.g\right|_{U_{\alpha}}=g_{\mathscr{L}_{\alpha}}=\operatorname{Hess} \mathscr{L}_{\alpha}$ for all $\alpha$. The triple $(M, S, g)$ will be called a locally Lagrange space or manifold.

It is easy to see that the local Lagrangians $\mathscr{L}_{\alpha}$ of a locally Lagrange space must again satisfy the compatibility relations (2.7), where the 1-forms $\varphi_{(\alpha \beta)}$ may not be closed. In particular, we see that a locally Lagrangian-symplectic manifold is a locally Lagrange space with the metric defined by [12]

$$
g([X],[Y])=\Omega(S X, Y),
$$

where $X, Y \in \Gamma T M$ and $[X]$, [Y] are the corresponding projections on $v \mathscr{F}$. Furthermore, if there exists a global Lagrangian $\mathscr{L}$ that is related by (2.7) with the local Lagrangians of the structure, $(M, S, g, \mathscr{L})$ will be a globally Lagrange space. A globally Lagrange space also is a globally Lagrangian-symplectic manifold, hence, it cannot be compact.

We can give a global characterization of the locally Lagrange metrics. First, we notice that the bundles $\otimes^{k} v^{*} \mathcal{V}$ of covariant tensors transversal to the vertical foliation $\mathscr{V}$ of a tangent manifold $(M, S)$ may also be seen as the bundles of covariant tensors on $M$ that vanish if evaluated on arguments one of which belongs to $\mathrm{im} S$. (This holds because $v^{*} \mathcal{V} \subseteq T^{*} M$ ). In particular, a transversal metric $g$ of $\mathscr{V}$ may be seen as a symmetric 2-covariant tensor field $g$ on $M$ which is annihilated by $\operatorname{im} S$. With $g$, one associates a 3-covariant tensor, called the derivative or Cartan tensor [1, 6, 7] defined by

$$
C_{x}(X, Y, Z)=\left(L_{S \tilde{X}} g\right)_{x}(Y, Z), \quad x \in M, X, Y, Z \in T_{x} M
$$


where $\tilde{X}$ is a foliated extension of $X$. Obviously, $C \in \Gamma \otimes^{3} v^{*} \mathcal{V}$. Then, we get the following proposition.

Proposition 2.3. The transversal metric $g$ of the vertical foliation $\mathcal{V}$ of a tangent manifold $(M, S)$ is a locally Lagrange metric if and only if the tensor field $C$ is totally symmetric.

Proof. Since

$$
C_{i j k}=C\left(\frac{\partial}{\partial x^{i}}, \frac{\partial}{\partial x^{j}}, \frac{\partial}{\partial x^{k}}\right)=\frac{\partial g_{j k}}{\partial y^{i}},
$$

the symmetry of $C$ is equivalent with the existence of the required local Lagrangians $\mathscr{L}$.

We give a number of examples of locally Lagrange manifolds.

EXAMPLE 2.4. Consider the torus of Example 1.6. Then

$$
\mathscr{L}=\frac{1}{2} \sum_{i=1}^{n}\left(y^{i}\right)^{2}
$$

define compatible local Lagrangians with the corresponding Lagrange metric $\sum_{i=1}^{n}\left(d x^{i}\right)^{2}$. (Notice also the existence of the locally Lagrange symplectic form $\Omega=\sum_{i=1}^{n} d x^{i} \wedge d y^{i}$.)

EXAMPLE 2.5. Consider the tangent manifold $M(1, p) \times(\mathbb{R} / \mathbb{Z})$ of Example 1.7 , with the tangent structure defined by (1.27). The $\mathscr{V}$-transversal metric

$$
\sum_{i=1}^{p}\left(d x^{i}\right)^{2}+(d y)^{2}
$$

is the Lagrange metric of the local compatible Lagrangians

$$
\frac{1}{2}\left(\sum_{i=1}^{p}\left(z^{i}\right)^{2}+t^{2}\right) .
$$

(In this example the forms $\varphi_{(\alpha \beta)}$ of (2.7) are not closed.)

Examples 2.4 and 2.5 are interesting because the manifolds involved are compact manifolds.

EXAMPLE 2.6. The manifold $M^{2 n}$ of Example 1.4 is diffeomorphic with the tangent bundle $T H^{n}$. With the coordinates $\left(x^{\prime i}, y^{\prime i}\right)$ (see Example 1.4), we see that the function

$$
\mathscr{L}=\frac{\sum_{i=1}^{n}\left(y^{\prime i}\right)^{2}}{2 \sum_{i=1}^{n}\left(x^{\prime i}\right)^{2}}
$$

is a global, regular Lagrangian, and it produces a positive definite Lagrange metric. 
EXAMPLE 2.7. Consider the Hopf manifold $H^{2 n}$ of Example 1.5 with the tangent structure (1.24), and define the local compatible Lagrangians

$$
\mathscr{L}=\frac{1}{2} \ln \rho, \quad \rho=\sum_{i=1}^{n}\left[\left(x^{i}\right)^{2}+\left(y^{i}\right)^{2}\right] .
$$

An easy computation yields

$$
\frac{\partial^{2} \mathscr{L}}{\partial y^{i} \partial y^{j}}=-\frac{1}{\rho^{2}}\left(2 y^{i} y^{j}-\rho \delta_{i j}\right)
$$

The determinant of the Hessian (2.16) can be easily computed as a characteristic polynomial, and we get

$$
\operatorname{det}\left(\frac{\partial^{2} \mathscr{L}}{\partial y^{i} \partial y^{j}}\right)=\frac{\sum_{i=1}^{n}\left[\left(x^{i}\right)^{2}-\left(y^{i}\right)^{2}\right]}{\left\{\sum_{i=1}^{n}\left[\left(x^{i}\right)^{2}+\left(y^{i}\right)^{2}\right]\right\}^{n+1}} \text {. }
$$

Now, the local equation

$$
\sum_{i=1}^{n}\left(x^{i}\right)^{2}=\sum_{i=1}^{n}\left(y^{i}\right)^{2}
$$

defines a global hypersurface $\Sigma$ of $H^{2 n}$, and (2.16) provides a locally Lagrange metric structure on $H^{2 n} \backslash \Sigma$.

EXAMPLE 2.8. On any tangent manifold $(M, S)$, any nondegenerate, foliated, transversal metric $g$ of the vertical foliation (if such a metric exists [8]) is locally Lagrange. Indeed, this kind of metric is characterized by $C=0$, and the result follows from Proposition 2.3.

A natural question implied by Definition 2.2 is: assume that $\left(M, S, g, \mathscr{L}_{\alpha}\right)$ is a locally Lagrange space; what are the conditions that ensure the existence of a global compatible, regular Lagrangian?

The compatibility relations (2.7) endow $M$ with an $\underline{A}^{0}$-valued 1-cocycle defined by any of the members of (2.7), hence, with a cohomology class $\mathscr{G} \in$ $H^{1}\left(M, \underline{A}^{0}\right)$, which we call the total Lagrangian obstruction. It is obvious that $\varphi=0$ if and only if the manifold $M$ with the indicated structure is a globally Lagrange space.

Furthermore, the total Lagrangian obstruction may be decomposed into two components determined by the exact sequence (1.7) with $k=0$, which in our case becomes

$$
0 \longrightarrow \Phi \stackrel{\subseteq}{\longrightarrow} \underline{A}^{0}(M, \mathscr{V}) \stackrel{\pi^{\prime}}{\longrightarrow} \underline{\Omega}_{p r}^{(1,0)} \longrightarrow 0,
$$

where $\pi^{\prime}$ is the composition of the projection $\pi$ of (1.7) by $S$. 
It is easy to see that the connecting homomorphism of the exact cohomology sequence of (2.19) is zero in dimension 0. Accordingly, we get the exact sequence

$$
0 \longrightarrow H^{1}(M, \Phi) \stackrel{\iota *}{\longrightarrow} H^{1}\left(M, \underline{A}^{0}\right) \stackrel{\pi *}{\longrightarrow} H^{1}\left(M, \underline{\Omega}_{p r}^{(1,0)}\right) \stackrel{\partial}{\longrightarrow} H^{2}(M, \Phi) \longrightarrow \cdots,
$$

where $\iota^{*}, \pi^{*}$ are induced by the inclusion and the homomorphism $\pi^{\prime}$ of (2.19). Accordingly, we get the cohomology class $\mathscr{G}_{1}=\pi^{*}(\mathscr{G}) \in H^{1}\left(M, \underline{\Omega}_{p r}^{(1,0)}\right)$, and we call it the first Lagrangian obstruction. The annulation $\mathscr{G}_{1}=0$ is a necessary condition for $M$ to be a globally Lagrange space. Furthermore, if $\varphi_{1}=0$, the exact sequence (2.20) tells us that there exists a unique cohomology class $\mathscr{G}_{2} \in$ $H^{1}(M, \Phi)$ such that $\mathscr{G}_{=} \iota^{*}\left(\mathscr{G}_{2}\right)$. We call $\mathscr{G}_{2}$ the second Lagrangian obstruction of the given structure, and $\mathscr{G}=0$ if and only if $\mathscr{G}_{1}=0$ and $\mathscr{G}_{2}=0$.

We summarize the previous analysis in the following proposition.

Proposition 2.9. The locally Lagrange space $\left(M, S, g, \mathscr{L}_{\alpha}\right)$ is a globally Lagrange space if and only if both the first and the second Lagrangian obstructions exist and are equal to zero.

Assume that a choice of a normal bundle $N^{\mathscr{V}}$ has been made. Then we can use the de Rham theorem associated with the relevant resolution (1.6) in order to get a representation of the Lagrangian obstructions. The definition of $\mathscr{G}_{1}$ shows that the first Lagrangian obstruction is represented by the cocycle $\left\{\theta_{\mathscr{L}_{\beta}}-\right.$ $\left.\theta_{\mathscr{L}_{\alpha}}\right\}$. Accordingly, $\mathscr{G}_{1}$ may be seen as the $d^{\prime \prime}$-cohomology class of the global form $\Theta$ of type $(1,1)$ defined by gluing up the local forms $\left\{d^{\prime \prime} \theta_{\mathscr{L}_{\alpha}}\right\}$. If we follow the notation of [9] and take bases

$$
N^{\mathscr{V}}=\operatorname{span}\left\{X_{i}=\frac{\partial}{\partial x^{i}}-t_{i}^{j} \frac{\partial}{\partial y^{j}}\right\}, \quad T^{\mathscr{V}}=\operatorname{span}\left\{Y_{i}=\frac{\partial}{\partial y^{i}}\right\},
$$

with the dual cobases

$$
\begin{aligned}
& N^{*} \mathscr{V}=\operatorname{ann}\left(T^{\mathscr{V}}\right)=\operatorname{span}\left\{d x^{i}\right\}, \\
& T^{* \mathscr{V}}=\operatorname{ann}\left(N^{\mathcal{V}}\right)=\operatorname{span}\left\{\mathcal{F}^{i}=d y^{i}+t_{j}^{i} d x^{j}\right\},
\end{aligned}
$$

where $t_{j}^{i}\left(x^{i}, y^{i}\right)$ are local functions, we get

$$
\Theta=\frac{\partial^{2} \mathscr{L}_{\alpha}}{\partial y^{i} \partial y^{j}} 9^{i} \wedge d x^{j}
$$

The result may be written as the following proposition.

Proposition 2.10. Let $\left(M, S, g, \mathscr{L}_{\alpha}\right)$ be a locally Lagrange space. Then, each choice of a normal bundle $N^{\mathcal{V}}$ defines an almost symplectic structure of $M$, given by the nondegenerate $d^{\prime \prime}$-closed 2 -form $\Theta$. The first Lagrangian obstruction $G_{1}$ vanishes if and only if the form $\Theta$ is $d^{\prime \prime}$-exact. 
COROLlary 2.11. A compact, connected, bundle-type, tangent manifold $M$, with the Euler vector field $E$ has no locally Lagrange metric $g$ such that $L_{E} g=s g$, where $s$ is a function such that, $\forall x \in M, s(x) \neq-1$.

Proof. Essentially, the hypothesis on $E$ means $E$ cannot be a conformal infinitesimal automorphism of $g$. From (2.23), we get

$$
\begin{aligned}
\Psi= & \frac{1}{n !} \Theta^{n}=(-1)^{n(n+1) / 2} \operatorname{det}\left(\frac{\partial^{2} \mathscr{L}_{\alpha}}{\partial y^{i} \partial y^{j}}\right) \\
& \cdot d x^{1} \wedge \cdots \wedge d x^{n} \wedge d y^{1} \wedge \cdots \wedge d y^{n} \\
L_{E} \Psi= & (-1)^{n(n+1) / 2}\left[E \operatorname{det}\left(\frac{\partial^{2} \mathscr{L}_{\alpha}}{\partial y^{i} \partial y^{j}}\right)+n \operatorname{det}\left(\frac{\partial^{2} \mathscr{L}_{\alpha}}{\partial y^{i} \partial y^{j}}\right)\right] \\
& \cdot d x^{1} \wedge \cdots \wedge d x^{n} \wedge d y^{1} \wedge \cdots \wedge d y^{n},
\end{aligned}
$$

where the local coordinates belong to an affine atlas such that $E=y^{i}\left(\partial / \partial y^{i}\right)$. If $M$ is compact, $\int_{M} L_{E} \Psi=0$, and the coefficient of the right-hand side of (2.25) cannot have a fixed sign. But the latter property holds under the hypothesis of the corollary.

For instance, the Hopf manifold $H^{n}$ has no locally Lagrange metric with homogeneous with respect to the coordinates $\left(y^{i}\right)$ Lagrangians $\mathscr{L}_{\alpha}$. Indeed, homogeneity of degree $s \neq-1$ is impossible because of Corollary 2.11, and homogeneity of degree -1 contradicts the transition relations (2.7).

REMARK 2.12. Because of Corollary 2.11, we conjecture that a compact, bundle-type, tangent manifold cannot have a locally Lagrange metric.

Proposition 2.13. The first Lagrangian obstruction of a locally Lagrange metric structure of $M$ with the local Lagrangians $\left\{\mathscr{L}_{\alpha}\right\}$ vanishes if and only if there exists a subordinated structure $\left\{\tilde{\mathscr{L}}_{\alpha}\right\}$ such that the 1-forms $\theta_{\tilde{L}_{\alpha}}$ glue up to a global 1-form. This subordinated structure defines a locally Lagrangiansymplectic structure on the manifold M. Furthermore, in this case the second Lagrangian obstruction $\varphi_{2}$ is represented by the global $d^{\prime \prime}$-closed form $\kappa$ of type $(0,1)$ defined by gluing up the local forms $\left\{d^{\prime \prime} \tilde{\mathscr{L}}_{\alpha}\right\}$.

Proof. Under the hypothesis, there exists a global form $\lambda$ of type $(1,0)$ such that $\Theta=d^{\prime \prime} \theta_{\mathscr{L}_{\alpha}}=d^{\prime \prime} \lambda$, therefore, $\theta_{\mathscr{L}_{\alpha}}=\left.\lambda\right|_{U_{\alpha}}+\xi_{\alpha}$, with some local foliated 1 -forms $\xi_{\alpha}=\xi_{\alpha, i}\left(x^{j}\right) d x^{i}$. Accordingly, we get

$$
\frac{\partial\left(\mathscr{L}_{\beta}-\mathscr{L}_{\alpha}\right)}{\partial y^{i}}=\xi_{\beta, i}-\xi_{\alpha, i}
$$

whence

$$
\mathscr{L}_{\beta}-\mathscr{L}_{\alpha}=a\left(\xi_{\beta}-\xi_{\alpha}\right)+b_{(\alpha \beta)},
$$

where $a$ has the same meaning as in (2.7) and $b_{(\alpha \beta)}$ are foliated functions. 
Now, if we define

$$
\tilde{\mathscr{L}}_{\alpha}=\mathscr{L}_{\alpha}-a\left(\xi_{\alpha}\right),
$$

we are done. The last assertion follows from the definition of $\varphi_{2}$.

COROLLARY 2.14. The locally Lagrange metric of Proposition 2.13 is defined by a global Lagrangian if and only if $\kappa=d^{\prime \prime} k$ for a function $k \in C^{\infty}(M)$.

In order to give an application of this result we recall the following lemma.

LEMMA 2.15. For the vertical foliation $\mathcal{V}$ of a tangent bundle $T N$, one has $H^{k}(T N, \Phi)=0$ for any $k>0$.

PROoF. Use a normal bundle $N^{\mathscr{V}}$, and let $\lambda$ be a $d^{\prime \prime}$-closed form of type $(p, q)$ on $T N$. Since the fibers of $T N$ are contractible, if $N=\cup U_{\alpha}$ is a covering by small enough, $T N$-trivializing neighborhoods, we have $\left.\lambda\right|_{p^{-1}\left(U_{\alpha}\right)}=d^{\prime \prime} \mu_{\alpha}$ $(p: T N \rightarrow N)$ for some local forms $\mu_{\alpha}$ of type $(p, q-1)$. The local forms $\mu_{\alpha}$ can be glued up to a global form $\mu$ by means of the pullback to $T N$ of a partition of unity on $N$, that is, by means of foliated functions. Accordingly, we will have $\lambda=d^{\prime \prime} \mu$.

From Corollary 2.14 and Lemma 2.15, we get the following proposition.

Proposition 2.16. Any locally Lagrange metric of a tangent bundle $T N$ is a globally Lagrange metric.

REMARK 2.17. Propositions 2.3 and 2.16 imply that, in the case of a tangent bundle $M=T N$, the symmetry of $C$ is a necessary and sufficient condition for $g$ to be a global Lagrangian metric. It was well known that this condition is necessary [6]. On the other hand, the metrics of [6] are usually differentiable only on the complement of the zero section of $T N$, where Proposition 2.16 does not hold, hence, the condition is not a sufficient one.

We also mention the inclusion $\sigma: \underline{Z}_{p r}^{(1,0)} \rightarrow \underline{\Omega}_{p r}^{(1,0)}$, where $Z$ denotes spaces of closed forms and the obvious following proposition.

Proposition 2.18. The locally Lagrange metric structure defined by $\left\{\mathscr{L}_{\alpha}\right\}$ is reducible to a locally Lagrangian-symplectic structure if and only if $\varphi_{1} \in \operatorname{im} \sigma^{*}$, where $\sigma^{*}$ is induced by $\sigma$ in cohomology.

Other important notions are defined by the following definition.

DEFINITION 2.19. Let $(M, S, g)$ be a locally Lagrange space, and let $X \in \Gamma T M$. Then: (i) $X$ is a Lagrange infinitesimal automorphism if $L_{X} g=0$, where $g$ is seen as a 2-covariant tensor field on $M$; (ii) $X$ is a strong Lagrange infinitesimal automorphism if it is a Lagrange and a tangential infinitesimal automorphism of $(M, S)$, simultaneously. 
Notice that

$$
\left(L_{X} g\right)(Y, S Z)=-g(Y,[X, S Z]) \quad(X, Y, Z \in \Gamma T M)
$$

From (2.29) and the nondegeneracy of $g$ on $v^{\mathscr{V}}$, it follows that a Lagrange infinitesimal automorphism is necessarily a $\mathscr{V}$-projectable vector field. But it may not be leafwise locally affine. Indeed, if $g$ is a foliated metric of $v^{\mathscr{V}}$ (Example 2.8), every tangent vector field of $\mathscr{V}$ is a Lagrange infinitesimal automorphism even if it is not leafwise locally affine.

We finish this section by considering a more general structure.

DEFINITION 2.20. Let $(M, S)$ be a tangent manifold. A locally conformal Lagrange structure on $M$ is a maximal open covering $M=\cup U_{\alpha}$ with local regular Lagrangians $\mathscr{L}_{\alpha}$ such that, over the intersections $U_{\alpha} \cap U_{\beta}$, the local Lagrangian metrics satisfy a relation of the form

$$
\mathcal{g}_{\mathscr{L}_{\beta}}=f_{(\alpha \beta)} \mathcal{g}_{\mathscr{L}_{\alpha}},
$$

where $f_{(\alpha \beta)}>0$ are foliated functions. A tangent manifold endowed with this type of structure is a locally conformal Lagrange space or manifold.

Clearly, condition (2.30) is equivalent with the transition relations

$$
\mathscr{L}_{\beta}=f_{(\alpha \beta)} \mathscr{L}_{\alpha}+a\left(\varphi_{(\alpha \beta)}\right)+b_{(\alpha \beta)},
$$

where the last two terms are like in (2.7). On the other hand, $\left\{\ln f_{(\alpha \beta)}\right\}$ is a $\Phi$-valued 1-cocycle, and may be written as $\ln f_{(\alpha \beta)}=\psi_{\beta}-\psi_{\alpha}$, where $\psi_{\alpha}$ is a differentiable function on $U_{\alpha}$ (which may be assumed projectable only if the cocycle is a coboundary). Accordingly, the formula

$$
\left.g\right|_{U_{\alpha}}=e^{-\psi_{\alpha}} g_{\mathscr{L}_{\alpha}}
$$

defines a global transversal metric of the vertical foliation which is locally conformal with local Lagrange metrics. As a matter of fact, we have the following proposition.

Proposition 2.21. Let $\left(M^{2 n}, S\right)$ be a tangent manifold, and let $n>1$. Then, $M$ is locally conformal Lagrange if and only if $M$ has a global transversal metric $g$ of the vertical foliation which is locally conformal with local Lagrange metrics.

Proof. We still have to prove that the existence of the metric $g$ that satisfies (2.32) implies (2.30), which is clear, except for the fact that the functions $f_{(\alpha \beta)}=e^{\psi_{\beta}-\psi_{\alpha}}$ are projectable. This follows from the Lagrangian character of 
the metrics $g_{\mathscr{L}_{\alpha}}$. Indeed, with the usual local coordinates $\left(x^{i}, y^{i}\right)$, the symmetry of the derivative tensors $C$ of $g_{\mathscr{L}_{\alpha}}, \mathfrak{g}_{\mathscr{L}_{\beta}}$ implies

$$
\frac{\partial f_{(\alpha \beta)}}{\partial y^{k}}\left(g_{\mathscr{L}_{\alpha}}\right)_{i j}=\frac{\partial f_{(\alpha \beta)}}{\partial y^{i}}\left(g_{\mathscr{L}_{\alpha}}\right)_{k j}
$$

and a contraction by $\left(g_{\mathscr{L}_{\alpha}}\right)^{i j}$ yields $\partial f_{(\alpha \beta)} / \partial y^{k}=0$.

The cohomology class $\eta=\left[\ln f_{(\alpha \beta)}\right] \in H^{1}(M, \Phi)$ will be called the complementary class of the metric $g$, and the locally conformal Lagrange metric $g$ is a locally Lagrange metric if and only if $\eta=0$. Indeed, if $\eta=0$, we may assume that the functions $\psi_{\alpha}$ are foliated and the derivative tensor $C$ of $g=e^{-\psi_{\alpha}} g_{\mathscr{L}_{\alpha}}$ is completely symmetric.

Furthermore, using a normal bundle $N^{\mathscr{V}}$ and the leafwise version of the de Rham theorem, the complementary class may be seen as the $d^{\prime \prime}$-cohomology class of the global, $d^{\prime \prime}$-closed complementary form $\tau$ obtained by gluing up the local forms $\left\{d^{\prime \prime} \psi_{\alpha}\right\}$. In particular, Lemma 2.15 and Proposition 2.16 imply that any locally conformal Lagrange metric $g$ of a tangent bundle must be a locally, therefore, a globally Lagrange metric.

EXAMPLE 2.22. Consider the Hopf manifold $H^{2 n}$ of Example 1.5. The local functions $\sum_{i=1}^{n}\left(y^{i}\right)^{2}$ define a locally conformal Lagrange structure on $H^{2 n}$, and

$$
g=\frac{\sum_{i=1}^{n}\left(y^{i}\right)^{2}}{\sum_{i=1}^{n}\left[\left(x^{i}\right)^{2}+\left(y^{i}\right)^{2}\right]}
$$

is a corresponding global metric, which, with the previously used notation, corresponds to

$$
\psi_{\alpha}=\ln \left\{\sum_{i=1}^{n}\left[\left(x^{i}\right)^{2}+\left(y^{i}\right)^{2}\right]\right\} .
$$

The corresponding complementary form is

$$
\tau=\frac{2 \sum_{i=1}^{n} y^{i} d y^{i}}{\sum_{i=1}^{n}\left[\left(x^{i}\right)^{2}+\left(y^{i}\right)^{2}\right]} .
$$

PROPOSITION 2.23. Let $(M, S)$ be a tangent manifold and $g$ a global transversal metric of the vertical foliation $\mathcal{V}$ of $S$. Then, $g$ is locally conformal Lagrange if and only if there exists a $d^{\prime \prime}$-closed form $\tau$ of type $(0,1)$ such that the tensor $\tilde{C}=C-(\tau \circ S) \otimes g$, where $C$ is the derivative tensor of $g$, is a completely symmetric tensor.

Proof. Define $\tilde{g}=e^{-\psi_{\alpha}} g$, where $\left.\tau\right|_{U_{\alpha}}=d^{\prime \prime} \psi_{\alpha}$ for a covering $M=\cup U_{\alpha}$. Then, $e^{-\psi_{\alpha}} \tilde{C}$ is the derivative tensor of $\tilde{g}$, and the result follows from Proposition 2.3. 
3. Transversal Riemannian geometry. The aim of this section is to give an index-free presentation of the connections used in Finsler and Lagrange geometry $[1,6,7]$, while also extending these connections to tangent manifolds.

Let $(M, S)$ be a tangent manifold and $g$ a metric of the transversal bundle of the vertical foliation $\mathscr{V}\left(T^{\mathscr{V}}=\mathrm{im} S\right)$. (The metrics which we consider are nondegenerate, but may be indefinite.) We do not get many interesting differentialgeometric objects on $M$, unless we fix a normal bundle $N^{\mathcal{V}}$, also called the horizontal bundle, that is, we decompose

$$
T M=N^{\mathscr{V}} \oplus T \mathscr{V}
$$

We will say that $N^{\mathscr{V}}$ is a normalization and $\left(M, S, N^{\mathscr{V}}\right)$ is a normalized tangent manifold. Where necessary, we will use the local bases (2.21) and (2.22). The projections on the two terms of (3.1) will be denoted by $p_{N}, p_{T}$, respectively, and $P=p_{N}-p_{T}$ is an almost product structure tensor that has the horizontal and vertical distribution as \pm 1 -eigendistributions, respectively.

For a normalized tangent manifold, the following facts are well known: (i) $\left.S\right|_{N \mathscr{V}}$ is an isomorphism $Q: N^{\mathscr{V}} \rightarrow T^{\mathscr{V}}$, (ii) $S=Q \oplus 0$, (iii) $S^{\prime}=0 \oplus Q^{-1}$ is an almost tangent structure, (iv) $F=S^{\prime}+S$ is an almost product structure, (v) $J=S^{\prime}-S$ is an almost complex structure on $M$.

On a normalized tangent manifold $\left(M, S, N^{\mathscr{V}}\right)$, a pseudo-Riemannian metric $\gamma$ is said to be a compatible metric if the subbundles $T^{\mathscr{V}}, N^{\mathscr{V}}$ are orthogonal with respect to $\gamma$ and

$$
\gamma(S X, S Y)=\gamma(X, Y), \quad \forall X, Y \in \Gamma N^{\mathcal{V}} .
$$

It is easy to see that these conditions imply the compatibility of $\gamma$ with the structures $J$ and $F$, that is,

$$
\gamma(J X, J Y)=\gamma(X, Y), \quad \gamma(F X, F Y)=\gamma(X, Y), \quad \forall X, Y \in \Gamma T M .
$$

Furthermore, if $(M, S)$ is a tangent manifold and $\gamma$ is a pseudo-Riemannian metric on $M$, we will say that $\gamma$ is compatible with the tangent structure $S$ if the $\gamma$-orthogonal bundle $N \mathcal{V}$ of $\operatorname{im} S$ is a normalization, and $\gamma$ is compatible for the normalized tangent manifold $\left(M, S, N^{\mathscr{V}}\right)$.

The following result is obvious.

Proposition 3.1. On a normalized, tangent manifold, any transversal metric $g$ of the vertical foliation defines a unique compatible metric $\gamma$ such that $\left.\gamma\right|_{N V}=g$.

In what follows, we will refer to the metric $\gamma$ as the canonical extension of the transversal metric $g$. On the other hand, a pseudo-Riemannian metric $\gamma$ of a tangent manifold $(M, S)$ which is the canonical extension of a locally Lagrange metric $g$ will be called a locally Lagrange-Riemann metric. This means that the restriction of $\gamma$ to the $\gamma$-orthogonal subbundle $N^{\mathcal{V}}$ of the vertical foliation $\mathscr{V}$ 
of $S$ is a locally Lagrange metric $g=g_{\mathscr{L}_{\alpha}}$ and $\gamma$ is compatible with $\left(M, S, N^{\mathcal{V}}\right)$. Then, $(M, S, \gamma)$ will be called a locally Lagrange-Riemann manifold. Notice that, since the induced metric of $N^{o} V$ is nondegenerate, $N^{*} V$ is a normalization of the vertical foliation and the compatibility condition of the definition makes sense. Thus, any normalized locally Lagrange space with the canonical extension $\gamma$ of the Lagrange metric $g$ is a locally Lagrange-Riemann manifold, and conversely.

EXAMPLE 3.2. The Euclidean metric $\sum_{i=1}^{n}\left[\left(d x^{i}\right)^{2}+\left(d y^{i}\right)^{2}\right]$ is the canonical extension of the locally Lagrange metric defined in Example 2.4 on the torus $\mathbb{T}^{2 n}$.

EXAMPLE 3.3. The metric

$$
\sum_{i=1}^{n}\left(d x^{i}\right)^{2}+(d y)^{2}+\sum_{i=1}^{n}\left(d z^{i}-x^{i} d y\right)^{2}+(d t)^{2}
$$

is the canonical extension of the locally Lagrange metric defined in Example 2.5 on $M(1, p) \times(\mathbb{R} / \mathbb{Z})$.

Now, let $\left(M, S, N^{\mathscr{V}}, g\right)$ be a normalized tangent manifold with a transversal metric of the vertical foliation $\mathscr{V}$ and let $\nabla$ be the Levi-Civita connection of the canonical extension $\gamma$ of $g$.

We are going to define a general connection that includes the connections used in Finsler and Lagrange geometry $[1,6,7]$ as particular cases determined by specific normalizations. This will be the so-called second canonical connection $D$ of a foliated, pseudo-Riemannian manifold $(M, \gamma)$, defined by the following conditions [9]: (i) $N^{\mathscr{V}}$ and $T \mathscr{V}$ are parallel, (ii) the restrictions of the metric to $N^{\mathscr{V}}$ and $T \mathscr{V}$ are preserved by parallel translations along curves that are tangent to $N^{\mathscr{V}}, T \mathscr{V}$, respectively, (iii) the $\mathscr{V}$-normal, respectively $\mathscr{V}$-tangent, component of the torsion $T_{D}(X, Y)$ vanishes if one of the arguments is normal, respectively tangent, to $\mathscr{V}$. This connection is given by

$$
\begin{aligned}
& D_{Z_{1}} Z_{2}=p_{N} \nabla_{Z_{1}} Z_{2}, \quad D_{Y_{1}} Y_{2}=p_{T} \nabla_{Y_{1}} Y_{2}, \\
& D_{Y_{1}} Z_{2}=p_{N}\left[Y_{1}, Z_{2}\right], \quad D_{Z_{1}} Y_{2}=p_{T}\left[Z_{1}, Y_{2}\right],
\end{aligned}
$$

where $Y_{1}, Y_{2} \in \Gamma T^{\mathscr{V}}$ and $Z_{1}, Z_{2} \in \Gamma N^{\mathscr{V}}$. We will say that $D$ is the canonical connection, and the connection induced by $D$ in the normal bundle $N^{\mathscr{V}}$, or, equivalently, in the transversal bundle $\mathcal{v} \mathscr{V}=T M / T \mathscr{V}$, will be called the canonical transversal connection. The canonical, transversal connection is a Bott (basic) connection [8]. The total torsion of the connection $D$ is not zero, namely, one has

$$
T_{D}(X, Y)=-p_{T}\left[p_{N} X, p_{N} Y\right], \quad \forall X, Y \in \Gamma T M
$$


Proposition 3.4. Let $(M, S, g)$ be a locally Lagrange manifold, and $\gamma$ the canonical extension of $g$. Then, the derivative tensor field of $g$ has the following expressions:

$$
\begin{aligned}
C(X, Y, Z) & =\left(D_{S X} \mathcal{g}\right)(Y, Z)=\left(D_{S X} \gamma\right)(Y, Z) \\
& =\gamma\left(\nabla_{Y}(S X), Z\right)+\gamma\left(Y, \nabla_{Z}(S X)\right),
\end{aligned}
$$

where $X, Y, Z \in \Gamma N^{\mathscr{V}}$.

Proof. Of course, in (3.7), $g$ is seen as a 2-covariant tensor field on $M$ (see Section 2). First, we refer to the first two equalities in (3.7). These are pointwise relations, hence, it will be enough to prove these equalities for foliated cross sections of the normal bundle $N^{\mathscr{V}}$. Indeed, a tangent vector at a point can always be extended to a projectable vector field on a neighborhood of that point. But in this case, the first and second equalities are straightforward consequences of the definitions of the tensor field $C$ and of the connection $D$. Then, since $\nabla$ has no torsion, (3.5) implies

$$
D_{S X} Y=\nabla_{S X} Y-p_{T} \nabla_{S X} Y-p_{N} \nabla_{Y}(S X)
$$

and, also using $\nabla \gamma=0$, we get the required result.

The first two expressions of $C$ actually hold for any vector fields $X, Y, Z \in$ ГTM.

COROLLARY 3.5. The canonical extension $\gamma$ of a transversal metric $g$ is a locally Lagrange-Riemann metric if and only if one of the following two equivalent relations holds:

$$
\begin{aligned}
\left(D_{S X} \gamma\right)(Y, Z) & =\left(D_{S Y} \gamma\right)(X, Z), \\
\gamma\left(\nabla_{Y}(S X), Z\right)+\gamma\left(Y, \nabla_{Z}(S X)\right) & =\gamma\left(\nabla_{X}(S Y), Z\right)+\gamma\left(X, \nabla_{Z}(S Y)\right),
\end{aligned}
$$

where $X, Y, Z \in \Gamma N \mathcal{V}$.

COROLlary 3.6. On a tangent manifold, if $\gamma$ is a compatible pseudoRiemannian metric such that $\nabla S=0$, then $\gamma$ is a projectable, locally LagrangeRiemann metric.

Proof. If $\nabla S=0$, the third equality (3.7) yields $C=0$, which is the characterization of this type of metrics.

Now, we consider the curvature of $D$. The curvature is a tensor and it suffices to evaluate it pointwisely. For this reason, whenever we need an evaluation of the curvature (as well as of any other tensor) that involves vector fields, it will suffice to make that evaluation on $\mathscr{V}$-projectable vector fields. 
Proposition 3.7. The curvature $R_{D}$ of the canonical connection has the following properties:

$$
\begin{aligned}
& R_{D}(S X, S Y) Z=0, \\
& R_{D}(S X, Y) Z=p_{N}\left[S X, D_{Y} Z\right], \\
& R_{D}(X, Y)(S Z)=-D_{S Z}\left(p_{T}[X, Y]\right), \\
& R_{D}(S X, Y) Z=R_{D}(S X, Z) Y,
\end{aligned}
$$

for any foliated vector fields $X, Y, Z \in \Gamma N^{\circ}$. Moreover, formulas (3.10), (3.12), and (3.13) hold for any arguments $X, Y, Z \in \Gamma N^{\mathcal{V}}$.

Proof. Equality (3.10) is in agreement with the fact that $D$ is a Bott connection [8]. Formulas (3.10), (3.11), and (3.12) follow from (3.5) and (3.6). Formula (3.13) is a consequence of (3.11). In the computation, one will take into account the fact that for any foliated vector field $X \in \Gamma T M$ and any vector field $Y \in \Gamma T \mathscr{V}$ one has $[X, Y] \in \Gamma T^{\circ} \mathscr{V}[8]$.

Proposition 3.8. For the canonical connection D, the first Bianchi identity is equivalent to the following equalities:

$$
\begin{gathered}
\sum_{\operatorname{Cycl}(X, Y, Z)} R_{D}(S X, S Y)(S Z)=0, \\
R_{D}(S X, Z) S Y=R_{D}(S Y, Z) S X, \\
\sum_{\operatorname{Cycl}(X, Y, Z)} R_{D}(X, Y) Z=0,
\end{gathered}
$$

where $X, Y, Z \in \Gamma N^{\mathscr{V}}$.

Proof. Write down the general expression of the Bianchi identity of a linear connection with torsion (e.g., $[4,5]$ ) for arguments tangent and normal to $\mathscr{V}$. Then, compute using (3.5), (3.6), and projectable vector fields as arguments. The fourth relation included in the Bianchi identity reduces to (3.12).

Proposition 3.9. For the canonical connection D, the second Bianchi identity is equivalent to the following equalities, where $X, Y, Z \in \Gamma N^{\mathcal{V}}$,

$$
\begin{gathered}
\sum_{\operatorname{Cycl}(X, Y, Z)}\left(D_{S X} R_{D}\right)(S Y, S Z)=0 \\
\left(D_{S X} R_{D}\right)(S Y, Z)-\left(D_{S Y} R_{D}\right)(S X, Z)=\left(D_{Z} R_{D}\right)(S X, S Y), \\
\left(D_{X} R_{D}\right)(Y, S Z)-\left(D_{Y} R_{D}\right)(X, S Z)+\left(D_{S Z} R_{D}\right)(X, Y)=R_{D}\left(p_{T}[X, Y], S Z\right), \\
\sum_{\operatorname{Cycl}(X, Y, Z)}\left(D_{X} R_{D}\right)(Y, Z)=\sum_{\operatorname{Cycl}(X, Y, Z)} R_{D}\left(p_{T}[X, Y], Z\right) .
\end{gathered}
$$

Proof. This is just a rewriting of the classical second Bianchi identity $[4,5]$ that uses (3.6). 
Like Riemannian geometry, we also define a covariant curvature tensor

$$
R_{D}(X, Y, Z, U)=\gamma\left(R_{D}(Z, U) Y, X\right), \quad X, Y, Z, U \in \Gamma T M .
$$

In particular, we have the following proposition.

Proposition 3.10.

$$
\begin{aligned}
R_{D}(U, Z, S X, Y) & =g\left(\left[S X, D_{Y} Z\right], U\right) \\
& =(S X)\left(g\left(D_{Y} Z, U\right)\right)-C\left(X, D_{Y} Z, U\right),
\end{aligned}
$$

where the arguments are foliated vector fields in $\Gamma N^{\mathscr{V}}$, and $g$ is seen as a tensor on $M$.

Formula (3.16) yields the Bianchi identity

$$
\sum_{\operatorname{Cycl}(X, Y, Z)} R_{D}(U, X, Y, Z)=0, \quad \forall X, Y, Z \in \Gamma N^{\mathscr{V}} .
$$

But the other Riemannian symmetries may not hold. Indeed, we have

Proposition 3.11. For any arguments $X, Y, Z, U \in \Gamma N^{\mathscr{V}}$,

$$
\begin{aligned}
R_{D}(X, Y, Z, U)+R_{D}(Y, X, Z, U) & =\left(D_{p_{T}[Z, U]} \gamma\right)(X, Y) \\
& =C\left(S^{\prime} p_{T}[Z, U], X, Y\right) .
\end{aligned}
$$

PROoF. Express the equality

$$
(Z U-U Z-[Z, U])(\gamma(X, Y))=0
$$

for normal foliated arguments, and use the transversal metric character of the canonical connection $D$ and Proposition 3.4.

Proposition 3.12. For any arguments $X, Y, Z, U \in \Gamma N^{\mathscr{V}}$,

$$
\begin{aligned}
R_{D}(X, Y, Z, U)-R_{D}(Z, U, X, Y) & \\
\quad= & \frac{1}{2}\left\{C\left(S^{\prime} p_{T}[Z, U], X, Y\right)-C\left(S^{\prime} p_{T}[X, Y], Z, U\right)\right\} .
\end{aligned}
$$

Proof. Same proof as for [4, Proposition 1.1, Chapter V].

The other first and second Bianchi identities may also be expressed in a covariant form. From (3.21), we get

$$
\left(D_{F} R_{D}\right)(A, B, C, E)=\gamma\left(\left(D_{F} R_{D}\right)(C, E) B, A\right)+\left(D_{F} \gamma\right)\left(R_{D}(C, E) B, A\right),
$$

where $(A, B, C, E, F \in \Gamma T M)$. Accordingly, (3.19) yields

$$
\begin{aligned}
\left(D_{S Z} R_{D}\right)(V, U, X, Y)+\left(D_{X} R_{D}\right)(V, U, Y, S Z)-\left(D_{Y} R_{D}\right)(V, U, X, S Z) \\
=\left(D_{S Z} \gamma\right)\left(R_{D}(X, Y) U, V\right)-\left(D_{X} \gamma\right)\left(p_{N}\left[S Z, D_{Y} U\right], V\right) \\
\quad+\left(D_{Y} \gamma\right)\left(p_{N}\left[S Z, D_{X} U\right], V\right),
\end{aligned}
$$


(3.20) yields

$$
\begin{aligned}
& \sum_{\operatorname{Cycl}(X, Y, Z)}\left(D_{X} R_{D}\right)(V, U, Y, Z) \\
& \quad=\sum_{\operatorname{Cycl}(X, Y, Z)} R_{D}\left(V, U, p_{T}[X, Y], Z\right)-\sum_{\operatorname{Cycl}(X, Y, Z)}\left(D_{X} \gamma\right)\left(R_{D}(Y, Z) U, V\right),
\end{aligned}
$$

where $X, Y, Z, U, V \in \Gamma N^{\circ} \mathcal{V}$, and so on.

EXAMPLE 3.13. On the torus $\mathbb{T}^{2 n}$ with the metric of Example 3.2, the usual flat connection is both the Levi-Civita connection and the canonical connection $D$, and it has zero curvature. On the manifold $M(1, p) \times(\mathbb{R} / \mathbb{Z})$ with the metric of Example 3.3, the connection that parallelizes the orthonormal basis shown by the expression of the metric is not the Levi-Civita connection, since it has torsion, but it follows easily that it has the characteristic properties of the canonical connection $D$. Accordingly, we are in the case of a locally LagrangeRiemann manifold with a vanishing curvature $R_{D}$ and a nonvanishing torsion $T_{D}$.

Proposition 3.14. The Ricci curvature tensor $\rho_{D}$ of the connection $D$ is given by the equalities

$$
\begin{aligned}
\rho_{D}(S X, S Y) & =\sum_{i=1}^{n}\left\langle\vartheta^{i}, R_{D}\left(\frac{\partial}{\partial y^{i}}, S X\right) S Y\right\rangle, \\
\rho_{D}(S X, Y) & =\sum_{i=1}^{n}\left\langle d x^{i}, p_{N}\left[D_{X_{i}} Y, S X\right]\right\rangle, \\
\rho_{D}(X, Y) & =\operatorname{tr}\left[Z \longmapsto R_{D}(Z, X) Y\right] \\
& =\sum_{i=1}^{n}\left\langle d x^{i}, R_{D}\left(X_{i}, X\right) Y\right\rangle,
\end{aligned}
$$

where $X, Y, Z \in \Gamma N^{\mathscr{V}}$, and in (3.31) $Y$ is projectable.

Proof. The definition of the Ricci tensor of a linear connection (e.g., [4]), and the use of the bases (2.21) and (2.22) yield

$$
\rho_{D}(X, Y)=\sum_{i=1}^{n}\left\langle d x^{i}, R_{D}\left(X_{i}, X\right) Y\right\rangle+\sum_{i=1}^{n}\left\langle 9^{i}, R_{D}\left(\frac{\partial}{\partial y^{i}}, X\right) Y\right\rangle .
$$

Then, the results follow from (3.5) and (3.11).

REMARK 3.15. In view of (3.32), we may speak of $\kappa_{D}=\operatorname{tr} \rho_{D}$ on $N^{\mathscr{V}}$, and call it the transversal scalar curvature.

In the case of a normalized, bundle-type, and tangent manifold $\left(M, S, E, N^{\mathscr{V}}\right)$, with a compatible metric $\gamma$ ( $E$ is the Euler vector field), the curvature has some more interesting features, which were studied previously in Finsler geometry [1]. These features follow from the following lemma. 
LEMMA 3.16. For any $Z \in \Gamma N \mathcal{V}$,

$$
D_{S Z}\left(S^{\prime} E\right)=Z \text {. }
$$

Proof. The tensor $S^{\prime}$ is the tensor defined at the beginning of this section, and with local bundle-type coordinates $\left(x^{i}, y^{i}\right)_{i=1}^{n}$ and bases (2.21), we have

$$
S Z=\xi^{i}\left(x^{j}, y^{j}\right) \frac{\partial}{\partial y^{i}}, \quad S^{\prime} E=y^{i} X_{i}
$$

Now, (3.34) follows from (3.5).

Using Lemma 3.16 one can prove the following proposition.

Proposition 3.17. The curvature operator $\left.R_{D}(X, Y)\right|_{N V}\left(X, Y \in \Gamma N^{\sigma}\right)$ is determined by its action on $S^{\prime} E$ and by $\left.R_{D}(V, S W)\right|_{N \mathscr{V}}$, where $V, W \in \Gamma N^{\circ}$.

Proof. Denote

$$
r(X, Y)=R_{D}(X, Y) S^{\prime} E .
$$

The covariant derivative of this tensor contains a term, which, in view of (3.34), is equal to $R_{D}(X, Y) Z$, and we get

$$
R_{D}(X, Y) Z=D_{S Z}(r(X, Y))-r\left(D_{S Z} X, Y\right)-r\left(X, D_{S Z} Y\right)-\left(D_{S Z} R_{D}\right)(X, Y) S^{\prime} E .
$$

Now, if the last term of (3.37) is expressed by means of the Bianchi identity (3.19), one gets an expression of $R_{D}(X, Y) Z$ in terms of $r$ and $\left.R_{D}(V, S W)\right|_{N V}$ for various arguments $V, W$.

Notice that, by (3.11), the computation of $\left.R_{D}(V, S W)\right|_{N \vee}$ on normal arguments requires only a first order covariant derivative.

From Proposition 3.17, we also see that the curvature values $R_{D}(U, Z, X, Y)$ $\left(X, Y, Z, U \in \Gamma N^{\mathcal{V}}\right)$ are determined by the values $R_{D}\left(U, S^{\prime} E, X, Y\right)$ and of $R_{D}(U$, $V, W, S K)$ for convenient normal arguments. Therefore, it should be interesting to study manifolds, where $R_{D}\left(U, S^{\prime} E, X, Y\right)$ has a simple expression. If we fix a direction $\operatorname{span}\{U\}$ and a 2-dimensional plane $\sigma=\operatorname{span}\{X, Y\}(U, X, Y \in \Gamma N \mathcal{V})$, the formula

$$
k_{U}(\sigma)=\frac{R_{D}\left(U, S^{\prime} E, X, Y\right)}{\gamma\left(S^{\prime} E, X\right) \gamma(U, Y)-\gamma\left(S^{\prime} E, Y\right) \gamma(U, X)}
$$

defines an invariant, which we will call the $U$-sectional curvature of $\sigma$. The invariant $k_{U}(\sigma)$ is independent of $U$ if and only if

$$
R_{D}(X, Y) S^{\prime} E=k(\sigma)\left[\gamma\left(S^{\prime} E, X\right) Y-\gamma\left(S^{\prime} E, Y\right) X\right]
$$


where $k(\sigma)$ is a function of the point of $M$ and the plane $\sigma$ only. Furthermore, if $k(\sigma)=f(x), x \in M$, that is, $k(\sigma)$ is pointwise constant, (3.39) is a natural simple expression of the transversal curvature tensor.

On the other hand, we can generalize the notion of flag curvature, which is an important invariant in Finsler geometry [1]. Namely, a flag $\phi$ at a point $x \in M$ is a 2-dimensional plane $\phi \subseteq T_{x} M$ which contains the vector $E_{x}$. Such a flag is $\phi=\operatorname{span}\left\{E_{x}, X_{x}\right\}$, where $X_{x} \in N_{x} \mathscr{V}$ is defined up to a scalar factor, and following [1], the flag curvature is defined by

$$
k(\phi)=k(X)=\frac{R_{D}\left(X, S^{\prime} E, X, S^{\prime} E\right)}{g\left(S^{\prime} E, S^{\prime} E\right) g(X, X)-g^{2}\left(S^{\prime} E, X\right)} .
$$

If $g$ is not positive definite, the flag curvature may take infinite values.

Proposition 3.18. The flag curvature $k$ is pointwise constant if and only if

$$
R_{D}\left(X, S^{\prime} E, Y, S^{\prime} E\right)=f\left[g\left(S^{\prime} E, S^{\prime} E\right) g(X, Y)-g\left(S^{\prime} E, X\right) g\left(S^{\prime} E, Y\right)\right],
$$

where $f \in C^{\infty}(M)$. If the $U$-sectional curvature is independent of $U$ and pointwise constant, the flag curvature is pointwise constant too.

PROOF. For the first assertion, use $k(X+Y)=k(X)=k(Y)$. The second follows because, if $k(\sigma)=f(x)$, (3.39) implies

$$
R_{D}\left(U, S^{\prime} E, X, Y\right)=f(x)\left[\gamma\left(S^{\prime} E, X\right) \gamma(Y, U)-\gamma\left(S^{\prime} E, Y\right) \gamma(X, U)\right],
$$

which reduces to (3.41) for $Y=S^{\prime} E$.

REMARK 3.19. The curvature $R_{D}$ has more interesting properties in the case of a bundle-type, locally Lagrange manifold such that the metric tensor $g$ is homogeneous of degree zero with respect to the coordinates $y^{i}$. The invariant characterization of this situation is that the derivative tensor $C$ is symmetric, and such that

$$
i\left(S^{\prime} E\right) C=0 .
$$

Indeed, in this case, formulas (3.24) and (3.26) yield simpler symmetry properties if one of the arguments is $S^{\prime} E$. The Finsler metrics satisfy the homogeneity condition (3.43).

REMARK 3.20. On a locally Lagrange-Riemann manifold $(M, S, \gamma)$ there exist other geometrically interesting connections as well. One such connection is

$$
\nabla_{X}^{\prime} Y=p_{N}\left(\nabla_{X}\left(p_{N} Y\right)\right)+p_{T}\left(\nabla_{X}\left(p_{T} Y\right)\right) .
$$

The connection $\nabla^{\prime}$ preserves the vertical and horizontal distributions and the metric, but has a nonzero torsion. Then, we have the connections ${ }^{C} D,{ }^{C} \nabla^{\prime}$, which can be defined by using formulas (3.5) and (3.44) with the Levi-Civita 
connection $\nabla$ replaced by the Chern connection ${ }^{C} \nabla$, that is, the $\gamma$-metric, $J$ preserving connection that has a torsion with no component of $J$-type $(1,1)$ $\left(J=S^{\prime}-S\right)[4,5]$.

We finish by recalling the well-known fact [3, 6, 7] that global Finsler and Lagrange structures of tangent bundles have an invariant normalization. This normalization may be defined as follows.

Let $\mathscr{L}$ be the global Lagrangian function. Then the energy function

$$
\mathscr{E} \mathscr{L}=E \mathscr{L}-\mathscr{L}
$$

has a Hamiltonian vector field $X_{\mathscr{E}}$ defined by

$$
i\left(X_{\mathscr{E}}\right) \omega_{\mathscr{L}}=-d \mathscr{E}_{\mathscr{L}},
$$

where $\omega_{\mathscr{L}}$ is the Lagrangian symplectic form (2.5), which turns out to be a second order vector field. Accordingly, $L_{X_{\mathscr{\varepsilon}}} S$ is an almost product structure on $M$ (see Section 1), and $N_{\mathscr{E}} \mathscr{V}=\operatorname{im} H$, with $H$ defined by (1.29) is a canonical normal bundle of $\mathscr{V}$.

A locally Lagrangian structure $\left\{\mathscr{L}_{\alpha}\right\}$ on a bundle-type tangent manifold ( $M, S$, E) defines a global function (second order energy)

$$
\mathscr{E}^{\prime}=E^{2} \mathscr{L}_{\alpha}-E \mathscr{L}_{\alpha}
$$

but, generally, it has no global Hamiltonian vector field, and, even if such a field exists, is may not be a second order vector field.

ACKNOWLEDGments. Part of the work on this paper was done during a visit at the Erwin Schrödinger International Institute for Mathematical Physics, Vienna, Austria, October 1-10, 2002, in the framework of the program "Aspects of Foliation Theory in Geometry, Topology, and Physics.” The author thanks the organizers of the program, J. Glazebrook, F. Kamber, and K. Richardson, and the ESI and its director professor P. Michor for having made that visit possible.

\section{REFERENCES}

[1] D. Bao, S.-S. Chern, and Z. Shen, An Introduction to Riemann-Finsler Geometry, Graduate Texts in Mathematics, vol. 200, Springer-Verlag, New York, 2000.

[2] R. S. Clark and M. R. Bruckheimer, Tensor structures on a differentiable manifold, Ann. Mat. Pura Appl. (4) 54 (1961), 123-141.

[3] J. Kern, Lagrange geometry, Arch. Math. (Basel) 25 (1974), 438-443.

[4] S. Kobayashi and K. Nomizu, Foundations of Differential Geometry. Vol I, Interscience Publishers, New York, 1963.

[5] _ _ Foundations of Differential Geometry. Vol. II, Interscience Tracts in Pure and Applied Mathematics, no. 15, Interscience Publishers, New York, 1969. 
[6] R. Miron and M. Anastasiei, The Geometry of Lagrange Spaces: Theory and Applications, Fundamental Theories of Physics, vol. 59, Kluwer Academic Publishers, Dordrecht, 1994.

[7] _ _ Vector Bundles and Lagrange Spaces with Applications to Relativity, Balkan Society of Geometers Monographs and Textbooks, vol. 1, Geometry Balkan Press, Bucharest, 1997.

[8] P. Molino, Riemannian Foliations, Progress in Mathematics, vol. 73, Birkhäuser Boston, Massachusetts, 1988.

[9] I. Vaisman, Cohomology and Differential Forms, Pure and Applied Mathematics, vol. 21, Marcel Dekker, New York, 1973.

[10] _,$d_{f}$-Cohomology of Lagrangian foliations, Monatsh. Math. 106 (1988), no. 3, 221-244.

[11]_ Second order Hamiltonian vector fields on tangent bundles, Differential Geom. Appl. 5 (1995), no. 2, 153-170.

[12] _ Locally Lagrange-symplectic manifolds, Geom. Dedicata 74 (1999), no. 1, 79-89.

[13] _ Locally Lagrangian symplectic and Poisson manifolds, Rend. Sem. Mat. Univ. Politec. Torino 59 (2001), no. 1, 43-58.

Izu Vaisman: Department of Mathematics, University of Haifa, 31905 Haifa, Israel

E-mail address: vaisman@math. haifa.ac. i1 


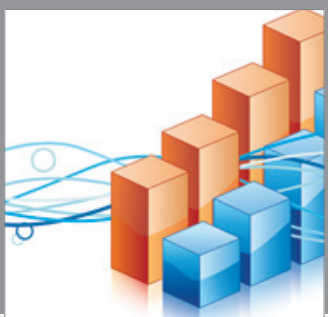

Advances in

Operations Research

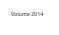

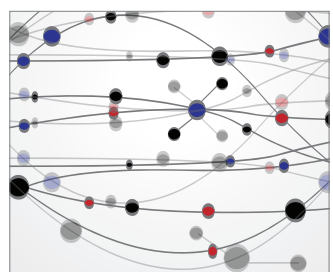

\section{The Scientific} World Journal
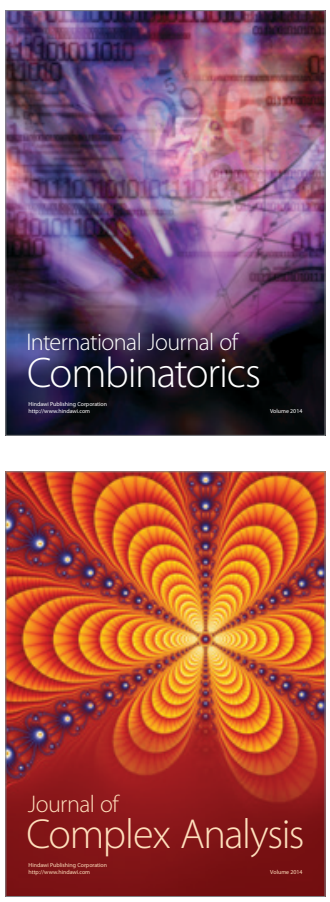

International Journal of

Mathematics and

Mathematical

Sciences
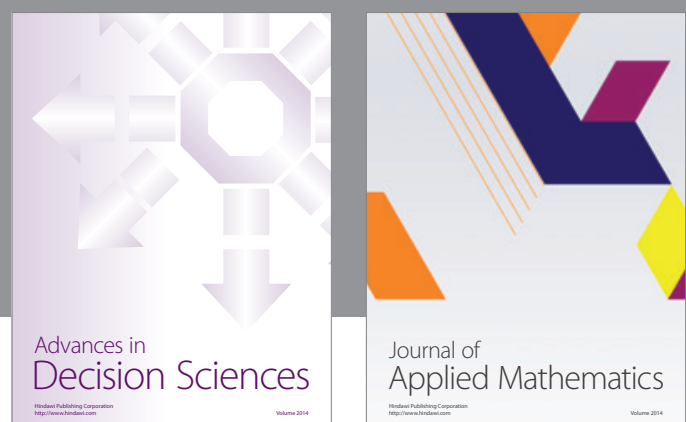

Journal of

Applied Mathematics
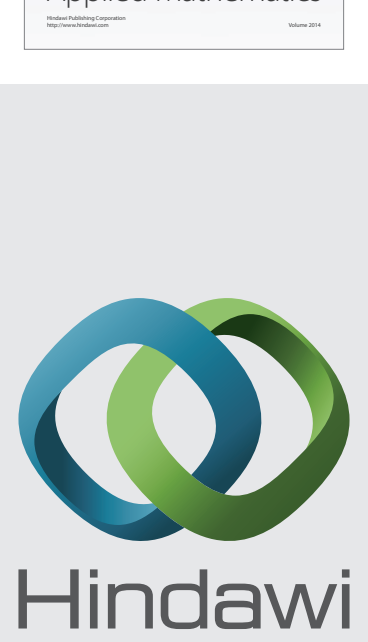

Submit your manuscripts at http://www.hindawi.com
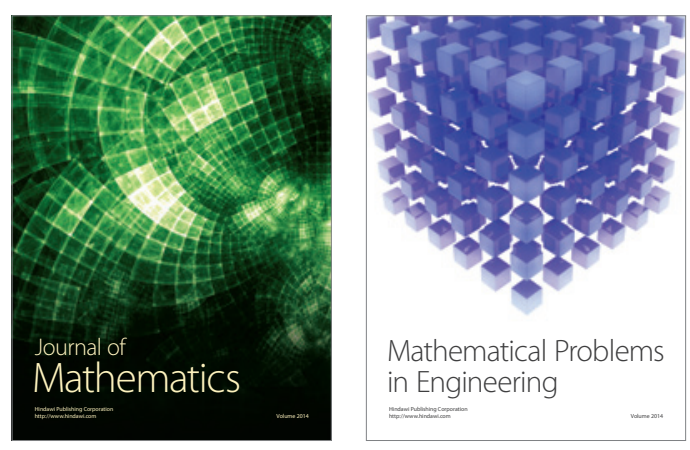

Mathematical Problems in Engineering
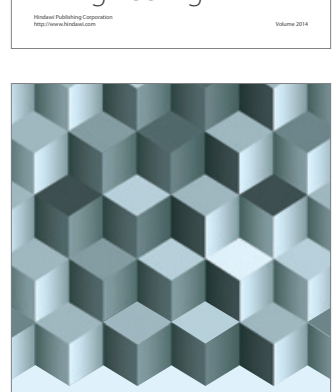

Journal of

Function Spaces
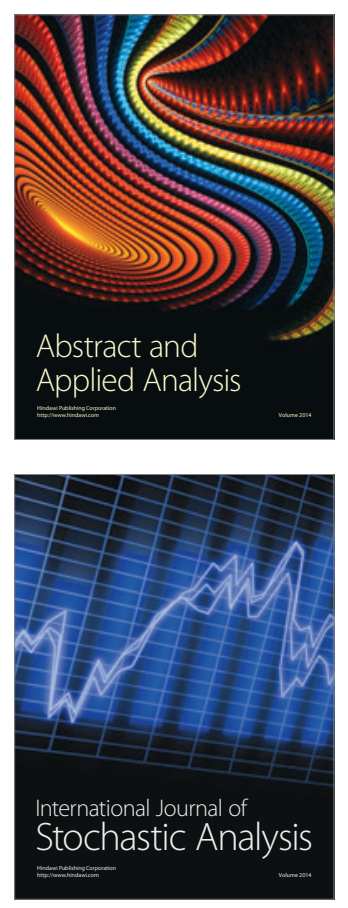

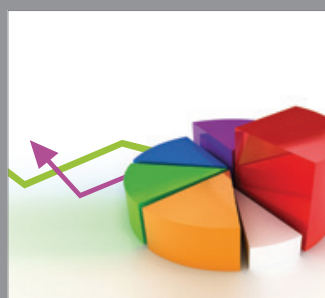

ournal of

Probability and Statistics

Promensencen
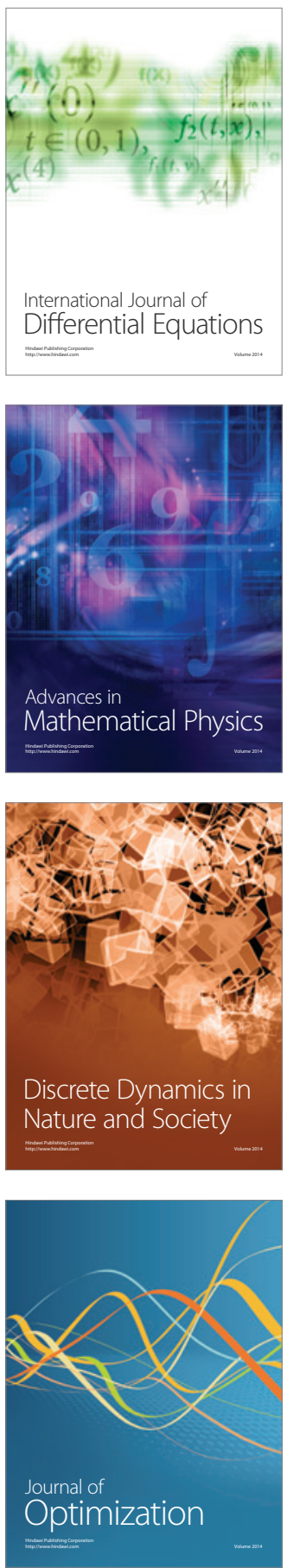